introduction of a new instrument that his ideas had already taken a concrete form. When one recalls the museum, which was probably the great feature of the London Meeting of the International Otological Congress, one is impressed with the desirability of building up a permanent one in connection with this Society. What a magnificent centre of attraction and instruction for the otologists of the United Kingdom.

Gentlemen, there never has been a time in the history of otology in this country when so many able, earnest, and scientific workers have been engaged in the investigation of its problems. Here we have in this Society over one hundred workers, many of them fired with the enthusiasm of youth, from whose earnest efforts we hopefully anticipate important results, which will have far-reaching and beneficent effects on the community.

\title{
LATENT OR INTERMITTENT NASAL OBSTRUCTION.
}

\author{
By Mayo Collier, M.B., M.S.Lond., F.R.C.S.Eng.,
}

Senior Surgeon to the North-West London Hospital ; and Ex-President of the British Laryngological Association, etc.

Mr. President and Gentlemen,-The object of my communication to-day is to ask you to consider with me a condition of things within the nose that up to the present has scarcely been entertained by rhinologists, and certainly not with the apprehension that this condition was one of any moment or worthy of much consideration. I want to point out to you that there is a condition of things with in the nose that is extremely common; that this condition, although extremely common, has escaped general recognition; that this condition of things is potent for harm, and is a source of many of the affections found within the throat, ear, nose, and post-nasal space.

The terms latent or intermittent nasal obstruction are sufficiently descriptive of the condition I wish to discuss with you this afternoon.

I suggest to you that, apart from the various forms of nasal obstruction due to growths, outgrowths, deflections, or what not, with which we are all familiar, there is a form of nasal obstruction that is more common and more harmful than all these put together.

1 Communicated to the British Laryngological, Rhinological, and Otological Association, January 29, 1901. 
This form of nasal obstruction is latent-that is to say, it may come on in a nose that is physiologically perfectly patent, and in which there is nothing abnormal to be seen. This form of obstruction may alternate with a condition of things in which the functions of the nose are perfectly performed, and yet for twelve hours out of the twenty-four the nose may be absolutely occluded, and the patient in a condition of extreme misery and discomfort.

This intermittent form of nasal obstruction often eludes recognition, and the surgeon is led astray because at the time of his examination the nasal chambers are perfectly free and patent, and the functions of the nose are perfectly performed.

I feel sure that no one present would resent the suggestion that more or less complete obstruction of the nasal chambers might be harmful to the individual, and might lead to ear, throat, or nose troubles. If you admit that constant nasal obstruction is in many cases a cause of ear, throat, and nose troubles, I am not straining rour imagination when I ask you to believe that intermittent nasal obstruction is also powerful for harm in the same direction. Or, in other words, if continuous loss of the functions of the nose results in trouble in the nose, throat, and ear, I am here to contend that intermittent loss of the functions of the nose has a similar effect.

This, then, is the subject matter of my remarks to you today. In order to properly understand this question we must have a clear idea of what are the functions of the nose and how the physiology of this organ is related to the physiology of the surrounding organs and to the economy in general. The nose has important relations with respiration, with digestion, with audition, with speaking and singing, with taste and smell, with sight, and with the various emotional states. The nose may also be looked upon as a respiratory organ in itself, as it is certain that a considerable interchange of gases takes place in its recesses and cavities. There is also some reason to believe that the nose is the safety valve of the brain.

In order to fully appreciate the effects of the loss of the functions of the nose and the harm that may in consequence accrue to the individual we must first be in a position to appreciate their value. This can only be done by a careful survey and accurate knowledge of these functions as far as we are able. In order to obtain this we will discuss seriatim the nose in relation to the various functions I have enumerated.

And first of all with respiration and so through respiration with the circulation and oxidation of the blood. As baldly stated in the 
physiology books, the nose warms, moistens, and filters the air, but how this is accomplished the writers of text-books have not attempted to explain. At first it is a little difficult to see how a stream of air some thirty or forty cubic inches in value can become warmed, moistened, and filtered whilst passing through the nose in the space of two seconds.

When, however, we examine the upper respiratory tract as a whole we see that it is not a channel or tube of one uniform calibre, but that it presents several remarkable constrictions that alternate with equally remarkable expansions.

We then begin to see that these constrictions prevent the very thing from taking place that we are told does take place, viz. that the air from the exterior passes directly through the nose from front to back and on into the lungs in a solid more or less cylindrical stream, and does not mix with its surroundings.

This is manifestly incorrect. If we look at the upper respiratory tract and view it as a whole, we shall be surprised at the variations that it presents.

Commencing with the external nares we find that the vestibule becomes narrower as it passes inwards and joins the nasal chamber opposite the nasal process of the superior maxillary bone. The vestibule or inlet to the respiratory function is cone shaped, and at its junction with the nasal chamber becomes so contracted as to constitute perhaps the narrowest point in the whole respiratory tract. It is curious to note that whereas the external nares are capable of expansion and contraction, the calibre of the upper respiratory tract at its junction with the nasal chamber is rigid and unalterable, being surrounded by bony and cartilaginous borders. A limit is thus put to nasal respiration by the size of the anterior opening of the nasal chamber.

After passing this narrow spot in the respiratory tract we find that the canal expands in a remarkable manner into the nasal chamber, which, with its recesses, constitutes a cavity of considerable dimensions. These recesses must not be dismissed from our calculations when studying the act of respiration. It is as sure and certain as anything can be that these recesses act not only as resonance chambers to the voice, but as warming and mixing chambers; so that the air as it is admitted from the exterior in a raw and unprepared state is mixed with the contents of the nose and its recesses, and so made ready to pass on into the pharynx and upper tubes.

An observation I had the advantage of making on a healthy frontal sinus will go a long way in substantiating this fact. 
I opened a frontal sinus for exploratory purposes, and being very careful I removed the button of bone without injury to the lining of the sinus. At each inspiration the lining membrane was depressed and at each expiration bulged into the wound, proving incontestibly that part of the contents of the nasal sinus is withdrawn during inspiration to be replaced during expiration.

The air, then, entering by the cone-shaped vestibule must do so with an increasing rate of speed till it has passed the narrows of the anterior opening. It must here lose much of its velocity, and, hecoming slowed down, will distribute itself in all directions, as regulated by the shape of the delivery tube of the vestibule.

It is thus seen that as a stream of air it must be subject to and conform to the shape of the tract through which it passes, and that the rate of motion of the air will be an average between the rates of motion in the narrowest and widest parts of the respiratory tract.

Passing onwards we come to a second marked narrowing - that of the posterior nares. This is crescent shaped, and varies with the size of the posterior end of the inferior turbinal body. The crossrection of this opening can only be a very small fraction of the cross-section of the nasal chamber.

Consequently the air in its passage hackwards must be greatly delayed by having to pass this constriction; thus allowing time for the necessary warming, moistening, and filtering of the inflow.

Following on this constriction is a remarkable dilatation, that of the pharynx, extending from the base of the skull to the upper opening of the larynx. The nasal contents having passed the posterior opening of the nares lingers in the pharynx and passes sowly on, but with increasing speed, to the larynx. The act of warming, moistening, and filtering is continued in the pharynx.

So far we have traced the inflowing air to the larynx, and can surmise that the anterior and posterior constrictions of the nasal chamber serve a very good purpose in delaying and slowing down the air in its passage through the nose. The narrow anterior' openmg or inlet insures that the inflowing air shall be distributed and mixed with the nasal contents. The posterior constriction prevents the too rapid emptying of the nasal chambers by the powerful aspiration of the lungs.

It is curious to note the alteration in the positions of the contents of the respiratory tract from the commencement of inspiration. At the commencement of inspiration the air in the bronchial tubes will pass in and fill the bronchioles and vesicles.

The air in the larynx and trachea will follow on into the 
bronchial tubes, and the air in the pharynx will pass on and take the place of the air in the trachea and large tubes, whilst the air in the nasal chambers will flow into the pharynx, larynx, and trachea. We thus see that the air passes in and mixes with and dilutes the impure reserve and residual air, but cannot displace it.

During respiration the impure expirate extrudes the contents of the pharynx and nose and fills the upper respiratory tract. This no doubt undergoes considerable purification in the chamber and recesses of the nose in the interval before the next inspiration.

I would here remind you whilst on the subject of respiration that this is the supreme function of the economy. The circulation and oxidation of the blood not only in the systemic, but portal system is dependent on respiration. It is not unreasonable to suggest to you that any interference with the function of respiration, and so with the proper oxidation and circulation of the blood, for a considerable period in the twenty-four hours must be harmful and baneful to the individual.

The functions of the nose are closely related to the functions of digestion, both directly and indirectly. Directly by the interference with mastication that follows an obstructed nose. A person with his nose occluded cannot shut his mouth sufficiently long to properly masticate his food, and consequently bolts the same before it is half divided, to the detriment of his stomach.

Again, the congestion of the pharynx and post-nasal space incidental to and associated with nasal obstruction upsets the stomach in more ways than one. Directly by the amount of unhealthy mucus that passes into the stomach, and indirectly by the constant irritation of the pneumogastric centre by the branches of the pharyngeal plexus.

The insanitary state of the mouth that follows mouth breathing, and the amount of contaminated and dusty mucus that must pass into the stomach (or what has been properly termed oral sepsis) ia considerable disadvantage to digestion.

Seeing that the whole of the circulation of the liver and abdominal organs is carried on by the respiratory act, any interference with this must be of serious detriment to the absorptive functions of the alimentary canal, and so a fertile source of slow digestion and other forms of dyspepsia.

Digestion and respiration are so intimately associated that I seldom meet with a patient suffering from nasal obstruction in a marked and chronic form who escapes from symptoms of indigestion, sluggish liver, or other abdominal troubles.

Now what relation have the functions of the nose to the eye? 
The nose is the drain of the conjunctival sac. Nasal obstruction will cause epiphora, and conjunctivitis, and other troubles. The two functions are intimately and sympathetically associated. The same nerve and the same artery supplies both. Any irritation in the nose is responded to by flushing of the conjunctiva and watering of the eye. The nose is the guardian of the eye. A strong light detrimental to the retina will cause sneezing, and so prevent further damage by altering the position of the head and casting the eyes to the ground, or closing them altogether. Congestion in the nose will be followed by fulness of the retinal veins and congestion of the conjunctiva. The nutrition of the nose and eye are intimately associated. The ophthalmic and spheno-palatine ganglia are anatomically and physiologically closely related.

Passing on to the relations of the nose to the ear, it is not surprising to find that these are peculiarly sensitive. The middle ear, or tubo-tympanum, is developed from the nose by two fingerlike prolongations. It is simply a prolongation of the nose cavity. The cavity of the nose and ear are practically one. Any increase or decrease of tension within the nose is immediately felt and communicated to the contents of the ear.

It is not difficult to realise that whatever affects the main cavity of the nose will affect its various recesses and prolongations. The unity of the nose and middle ear must be taken into consideration and fully realised as the first and essential step in the proper understanding and treatment of many of the affections of this culde-sac.

There is little doubt that in normal nasal respiration both inspiration and expiration are felt in the tympanic cavity, and that a slight movement of the membrana tympani takes place, inwards with inspiration and outwards with expiration, the same as has been recorded in the frontal sinus. In corroboration of this may be mentioned the fact that when one is listening very carefully for a sound the mouth is opened, and respiration is suspended or carried on very gently by way of the mouth, in order to annul-I *uggest-the oscillations incidental to respiration.

The relation between the functions of the nose and the voice are peculiarly apparent. Besides preserving the covering of this organ in a healthy condition, the nasal chamber is the delivery-tube of the roice, as well as its resonator.

The functions of smell and taste are almost entirely dependent on the patency and proper functioning of the nose, and must be entirely annulled if the nose be occluded.

And lastly, there are some grounds for supposing that the nose 
is the safety valve of the brain, by acting as a drain to the arachnoid cavity. We know that the arachnoid sheath is continued through each opening in the cribriform plate, and surrounds the olfactory nerves almost to their terminations. 'The confusion of intellect, the forgetfulness, the difficulty of concentrating the thoughts, the headache, the morning drowsiness, and other mental symptoms that are associated with nasal obstruction may well be due to increase of arachnoid fluid and congestion following on nasal obstruction and the consequent arrest of the functions of the nose. There may be some reason also for believing that some at least of the large quantity of moisture that is taken up by the inspired air comes from the arachnoid fluid. Again, the pathological conditions known as hydrorrhoea, where pints of fluid are discharged from the nasal cavities, associated with polypi and other abnormal states, can more easily be explained by the assumption of an outflow from the arachnoid extensions into the nose modified possibly by infiltration.

In order to remind you of the relation of the functions of the nose to the emotions I would ask you to imagine a great actor in any important part with an obstructed nose and an open mouth. The emotional display of the face would be quite impossible, and the actor's efforts ruined.

The relation of the nose to the sexual function is well marked in some of the carnivora, but may, I think, be altogether discarded when dealing with the genus homo.

Now, gentlemen, you will agree with me that the nose is not an unimportant or idle organ, and is well worthy of our best care and consideration.

I will now return to our main subject and explain to you more fully what is meant by latent or intermittent nasal obstruction, the mechanism of its production, its diagnosis, and lastly, if time allows, I shall say a few words as to treatment. I can more easily explain to you the exact condition to be known as latent or intermittent nasal obstruction by citing you one or more out of the large number of cases I have records of.

In 1895 I was consulted by a medical man, who gave the following account of himself:--He was sixty-three years of age; for the last fourteen or fifteen year's he had been troubled with his nose becoming occluded at night and at other times. His general health, he said, was indifferent: he was easily fatigued, and suffered from flatulent dyspepsia, headache, and deafness. His sleep was constantly disturbed at night, and he awoke in the morning unrefreshed and tired. He said for years he had suffered from 
catarrh in the nose and head, but lately things had become so much worse that he felt sure he would go out of his mind if something was not done for him. This gentleman was a tall, pale, liverish-looking person with suffering stamped on his face.

I ascertained that he had treated himself with washes, snuff, and inhalations, but with little effect. That he had been under the care of a distinguished physician, who assured him his heart, lungs, and abdominal organs were sound. I found in his present condition nothing that one could lay one's hands on and label as disease. The throat was pale and anæmic, the interior of the nose was pale, and the mucous membrane was if anything anæmic. There was ample room for nasal respiration, and nothing abnormal or irregular was apparent, save one sign, which I venture to suggest was the key to the whole situation. On either side of the septum there was the tell-tale hollow or groove, the exact cast of the lower turbinal body. Beyond this there was nothing to indicate that the nose had been anything but free night or day.

Well, gentlemen, with this physical condition of complete freedom of nasal respiration and the absence of anything abnormal with the nose, this patient was on the very verge of melancholia from the misery and discomfort of complete nasal occlusion so soon as his head was placed upon the pillow. The functions of the nose were here intermittently performed. For sixteen hours it was free, for eight hours it was closed. Yet this nocturnal occlusion was quite sufficient to affect and put out of gear all the correlated functions of the nose, and to bring the economy almost to a standstill.

An almost exactly similar case was that of a barrister sent to me from Dublin by a member of this Association. These are extreme but typical cases, and happily, I am glad to say, are few and far between.

The commoner forms of intermittent nasal obstruction present symptoms of less severity. In many cases there is only the slight hoarseness and morning cough and difficulty in clearing the throat on rising in the morning. There may only be a tendency to flatulent dyspepsia or distension after meals. The hearing may not be quite so good as it was, and the patient may tell you he thinks he is getting deafer, until he finds out he is suffering from a marked degree of deafness. This is constantly the only symptom of intermittent nasal obstruction.

I believe that in intermittent or latent nasal obstruction we have a valid and sufficient explanation for the existence of that large class of slow, insidious, painless forms of ear trouble known as 
chronic progressive deafness. Given an occlusion of the nose for eight out of the twenty-four hours, and you have all the factors ready and able to produce occlusion of the Eustachian tubes, congestion of the tympanic cavity, and depression of the drumhead, and the subsequent physical change known as sclerosis. This explanation is worthy of your consideration, and the more I live and learn, the more assured $\mathrm{I}$ am of its soundness.

I have on more than one occasion insisted that there is an intimate relation between intermittent nasal obstruction and the affections of the other recesses and extensions of the nose, and the same anatomical and physiological facts that govern the recess of the middle ear apply equally with all the other extensions of the nose. The tympanic cavity and the antrum or frontal sinus are on all fours with each other. They have a common origin, their lining membrane is similar and continuous. They are subject to the same variations in air tension and supply, and they are subject to the same affections.

I have recently exhibited to you a case of frontal-sinus trouble where the suppuration was kept up for three years by occlusion of the nostril on the same side from collapse of the wing of the nose and enlargement of the turbinal body. On reducing the turbinal body and supporting the wing, the suppuration promptly disappeared. Uncomplicated chronic suppurations of the accessory cavities of the nose are induced and maintained by intermittent obstructions of the nose. This applies equally to the aural accessory cavity. The obstinate discharges from these cavities that continue sometimes after operations are to be explained when intermittent nasal obstruction is present.

We have heard a good deal lately about the atiology and treatment of polypi. My experience is that when these growths are properly removed and the nose properly ventilated and nocturnal nasal obstruction prevented they do not return. I have one case that illustrates my meaning perfectly. I operated on a gentleman, whom I exhibited in this room as a typical martyr to asthma relieved by clearing his nose from polypi. Well, those growths recurred and required removal off and on for eleven years. In the earlier periods of my treatment I took no account of the fact that he was completely obstructed at night-time and yet was free during the day. The asthma was better when the nose was cleared, but returned with the obstruction. Some four or five years ago I operated on both lower turbinal bodies. I have never had occasion to treat him medically since, and his nose remains perfectly free from polypi to this day. I believe that intermittent nasal obstruc- 
tion plays an important part in the production and recurrence of polypi. Much more could I say on this important subject, but the time at my disposal will not suffice for a more extended view.

We pass now on to the subject of diagnosis. How do we know when intermittent nasal obstruction is present or absent? If there is nothing abnormal to be seen in the nose, and if the functions of nasal respiration are perfectly and adequately performed, by what signs shall we be able to recognise this form of obstruction? In latent or intermittent nasal obstruction the obstruction as a rule sccurs at night-time, when the head is on the pillow and the body is more or less in a horizontal position. When up and about, as in itting, walking, or standing, there is seldom obstruction, except on passing from a cold to a warm atmosphere, and sometimes when lrinking hot liquids or taking spiced or pungent meals and after alcoholic driuks. In the vertical position of the trunk, gravity retains the venous blood in the great vessels of the neck, chest, and abdomen. On assuming the horizontal position the blood flows into the veins and sinuses of the head and neck in response to the ame force. When sleeping the arteries become smaller in calibre and the veins larger, so that the veins of the head and neck contain relatively more blood. The cavernous tissue and mucous membrane of the nose becomes, when lax and atonic, distended mechanically by the increased intra-vascular pressure caused by the mere inflowing of blood to these parts. The distended turbinal hodies approach the septum on either side, and press upon it so vigorously as to form a groove or exact cast of themselves and rompletely obliterate the lumen of the nasal chambers. The erectile tissue on the floor and lower part of the septum helps to complete this. The groove then is to be sought for, and is unloubtedly evidence of the condition I am attempting to describe to you. It is indeed more than evidence, it is pathognomonic of intermittent nasal obstruction, and is the only objective sign that will present itself to you.

With subjective symptoms of laryngeal, throat, nose, or ear trouble and the objective sign I have described, you have all the evidence required to make the diagnosis of intermittent nasal obstruction. I place no credence on the answers of patients to the question as to the method of breathing at night. Some, very often the worst cases, will indignantly repudiate the suggestion that they breathe through the mouth at night, yet will in the same hreath tell you that they awake with the tongue dry, and are always anxious for a cup of tea in the morning.

The subject of treatment will involve much time and considera- 
tion, and as I feel I have already occupied a large measure of your time, and, like yourselves, am anxious and looking forward to the President's Address, I will postpone the subject of treatment to a more opportune moment.

If, in the remarks I have made, I have given you any suggestions worthy of your acceptance or set you thinking on this important subject, I shall be more than amply repaid for the anxiety and labour attendant on this communication.

\title{
THE AETIOLOGY AND TREATMENT OF LABYRINTHINE SUPPURATION.*
}

\author{
By Wiluiam Milligan, M.D.,
}

Surgeon to the Manchester Hospital for Diseases of the Ear; Aurnl Surgeon to the Manchester Royal Infirmary; Lecturer upon Diseases of the Ear, the University of Manchester.

Mr. President and Gentiman,- - Septic disease of the labyrinth demands our most careful consideration, in the first place on account of the damage which may be done to the acoustic or to the static segments of the internal ear, and in the second place on account of the risk which exists of the pathogenic process extending to the basal meninges or to the adjacent lobes of the cerebellum. The most frequent causes of infection of the internal ear are as follows:-(1) Suppurative lesions of the middle ear extending through the external labyrinthine wall; (2) suppurative processe: originating at the base of the brain and extending along the perivascular or perinenral sheaths of the auditory or facial nerves: (3) deposition of pathogenic organisms by the general bloodstream in some portion of the internal ear; (4) injuries.

The most frequent and, from the practical point of view, the most important cause is extension from the cavity of the middl' ear. In the great majority of cases due to this particular cause perforation into the labyrinth has taken place, either as the result of erosion of the bony labyrinthine wall or as the result of maceration of the fibrous structures uniting the foot-plate of the staper to the margins of the foramen ovale or of the membrana secundaria covering the fenestra rotunda.

Perforations leading into the cavity of the internal ear may

1 Paper read at the meeting of the Otolngical Society of the United Kingdom, February 1, 1904. 